\title{
Analyse De L'évolution Saisonnière Des Matières Oxydables Dans Le Secteur Oriental De La Lagune Ebrié (Côte d'Ivoire)
}

\author{
Irié B.T. Jean-Gaël \\ Wognin A. Valérie \\ Kando A. Marie-Laure
}

Département des Géosciences Marines, UFR STRM, Université Félix

Houphouët-Boigny, Abidjan, Côte d'Ivoire

Aka Natcha

Centre de Recherches Océanologiques (CRO), Abidjan, Côte d'Ivoire

Aka A. Maurice

Coulibaly A. Sougo

Monde Sylvain

Département des Géosciences Marines, UFR STRM, Université Félix

Houphouët-Boigny, Abidjan, Côte d'Ivoire

Doi: 10.19044/esj.2018.v14n3p221 URL:http://dx.doi.org/10.19044/esj.2018.v14n3p221

\begin{abstract}
Faced with the extension of the cities of Grand-Bassam and Abidjan along the Ebrie lagoon and the lack of data on the oxidizable content in the eastern sector of the Ebrié lagoon, this study was initiated. It aims to determine the levels of oxidizable materials in the eastern sector of the Ebrié lagoon. PH, temperature, salinity and dissolved oxygen were determined in situ at thirtyone (31) stations during the dry season and the rainy season. On these same stations, water samples were also taken per season to determine the content of oxidizable matter according to the methods in force. The statistical treatment consisted in knowing the links between the hydrological parameters. The average values of the parameters in the rainy season and in the dry season are 5,98 and 7,28 respectively for the $\mathrm{pH}, 27,59{ }^{\circ} \mathrm{C}$ and $29,6{ }^{\circ} \mathrm{C}$ for the temperature, 5,01 and 19,48 for salinity, $6.14 \mathrm{mg} / \mathrm{L}$ and $3.78 \mathrm{mg} / \mathrm{L}$ for dissolved oxygen, $0.24 \mathrm{mg} / \mathrm{L}$ and $0.38 \mathrm{mg} / \mathrm{L}$ for ammonium, $0.089 \mathrm{mg} / \mathrm{L}$ and $0.036 \mathrm{mg} / \mathrm{L}$ for nitrite, $8.18 \mathrm{mg} / \mathrm{L}$ and $0.23 \mathrm{mg} / \mathrm{L}$ for nitrate, $1.57 \mathrm{mg} / \mathrm{L}$ and $1.78 \mathrm{mg} / \mathrm{L}$ for orthophosphate, $76.06 \mathrm{mg} / \mathrm{L}$ and $56.81 \mathrm{mg} / \mathrm{L}$ for COD and 40.23 $\mathrm{mg} / \mathrm{L}$ and $34.16 \mathrm{mg} / \mathrm{L}$ for BOD 5 . The evolution of oxidizable matter depends on the entry of continental, oceanic and precipitation waters into the lagoon. The high levels of oxidizable materials are observed in large part in the berries.
\end{abstract}


Good positive correlations are observed between $\mathrm{COD}$ and $\mathrm{DBO}_{5}$ and between salinity and nitrite. Temperature and salinity have a good negative correlation. Lagoon waters receive more oxidizable materials in highly urbanized areas than in areas with little or no urbanization.

Keywords : Oxidizable materials, rainy and dry season, Ebrié lagoon

\section{Résumé}

Face à l'extension des villes de Grand-Bassam et d'Abidjan le long de la lagune Ebrié et au manque de données sur les teneurs en matières oxydables dans le secteur oriental de la lagune Ebrié, cette étude a été initiée. Elle a pour objectif de déterminer les teneurs des matières oxydables dans le secteur oriental de la lagune Ebrié. Le $\mathrm{pH}$, la température, la salinité et l'oxygène dissous ont été déterminés in situ au niveau de trente et un (31) stations pendant la saison sèche et la saison pluvieuse. Sur ces mêmes stations des échantillons d'eau ont également été prélevés par saison pour la détermination de la teneur des matières oxydables selon les méthodes en vigueur. Le traitement statistique a consisté à connaitre les liens entre les paramètres hydrologiques. Les valeurs moyennes des paramètres en saison pluvieuse et en saison sèche sont respectivement égales à 5,98 et 7,28 pour le $\mathrm{pH}, 27,59^{\circ} \mathrm{C}$ et $29,6^{\circ} \mathrm{C}$ pour la température, 5,01 et 19,48 pour la salinité, $6,14 \mathrm{mg} / \mathrm{L}$ et $3,78 \mathrm{mg} / \mathrm{L}$ pour l'oxygène dissous, $0,24 \mathrm{mg} / \mathrm{L}$ et $0,38 \mathrm{mg} / \mathrm{L}$ pour l'ammonium, $0,089 \mathrm{mg} / \mathrm{L}$ et $0,036 \mathrm{mg} / \mathrm{L}$ pour le nitrite, $8,18 \mathrm{mg} / \mathrm{L}$ et $0,23 \mathrm{mg} / \mathrm{L}$ pour le nitrate, $1,57 \mathrm{mg} / \mathrm{L}$ et $1,78 \mathrm{mg} / \mathrm{L}$ pour l'orthophosphate, $76,06 \mathrm{mg} / \mathrm{L}$ et $56,81 \mathrm{mg} / \mathrm{L}$ pour la $\mathrm{DCO}$ et à $40,23 \mathrm{mg} / \mathrm{L}$ et $34,16 \mathrm{mg} / \mathrm{L}$ pour la $\mathrm{DBO}_{5}$. L'évolution des matières oxydables dépend de l'entrée d'eaux continentales, océaniques et des eaux de précipitations dans la lagune. Les fortes teneurs en matières oxydables s'observent en grande partie dans les baies. Les bonnes corrélations positives s'observent entre la $\mathrm{DCO}$ et la $\mathrm{DBO}_{5}$ et entre la salinité et le nitrite. La température et la salinité ont une bonne corrélation négative. Les eaux de la lagune Ebrié sont de mauvaises qualités. Elles reçoivent plus de matières oxydables dans les zones fortement urbanisées que dans les zones peu ou non urbanisées.

Mots-clés : Matières oxydables, saison pluvieuse et sèche, lagune Ebrié

\section{Introduction}

Le littoral ivoirien est bordé de lagunes dont les principales sont les lagunes Ebrié, Aby et la lagune de Grand-Lahou. La lagune Ebrié a l'avantage d'être vaste $\left(523 \mathrm{~km}^{2}\right)$, facilement accessible et de présenter une grande variété d'espèces (Binder, 1968). Ce plan d'eau est soumis aux effets des rivières côtières, des précipitations, du fleuve Comoé et de l'océan atlantique à travers 
le canal de Vridi. Les influences de ces facteurs permettent de distinguer au niveau de la lagune Ebrié une partie Est ou secteur oriental et une partie Ouest (Durand et Guiral, 1994). Le secteur oriental objet de cette étude se situe entre la baie d'Adiapodoumé et le fleuve Comoé à Grand-Bassam. Il constitue une zone d'intérêt économique important du fait des activités industrielles, touristiques et portuaires. Les travaux antérieurs réalisés dans la lagune Ebrié ont porté essentiellement sur certaines baies de la zone estuarienne de la lagune Ebrié à cause de la ville d'Abidjan (Aka et Koné, 1996 ; Yao et al, 2009 ; Inza et al, 2009 ; Adingra et Kouassi, 2011, Aka et al, 2017a, Aka et al, 2017b). Cependant ils n'ont pas permis de connaitre les teneurs des matières oxydables au niveau du secteur oriental. Pourtant, ces dernières années, on assiste à une extension des villes de Grand-Bassam et d'Abidjan le long de la lagune Ebrié. A partir de ces constats, ce travail se donne pour objectif de déterminer la concentration des matières oxydables dans la région orientale de la lagune Ebrié. En outre, l'étude saisonnière permettra de connaitre l'influence des saisons sur l'évolution de chaque paramètre.

\section{Materiel et methodes}

\section{Mesure des paramètres hydrologiques}

Deux campagnes organisées en saison des pluies (juillet 2015) et en saison sèche (février 2016) ont permis de mesurer le potentiel d'hydrogène $(\mathrm{pH})$, la température $\left(\mathrm{T}^{\circ}\right)$, l'oxygène dissous $\left(\mathrm{O}_{2}\right)$ et de la salinité $(\mathrm{Sal})$ au niveau de trente-un (31) stations. Les mesures des paramètres sont faites à 0,5 mètre par un multiparamètre YSI V2. Les sondes du multiparamètre sont plongées directement dans l'eau pour les différentes mesures.

\section{Prélèvement des échantillons d'eau et analyse des matières oxydables}

Le prélèvement des échantillons d'eau se fait à l'aide d'une bouteille Niskin de capacité 2 litres. L'eau prélevée est conservée dans des flacons ou bouteilles appropriés pour être transportée au laboratoire pour les analyses. Un GPS de type Garmin est utilisé pour la détermination de la position des points d'échantillonnage. Les campagnes d'échantillonnage ont permis de visiter trente-un (31) stations (Figure 1) deux fois pendant la saison des pluies et la saison sèche. Les teneurs du nitrite $\left(\mathrm{NO}_{2}\right)$, du nitrate $\left(\mathrm{NO}_{3}\right)$, de l'ammonium $\left(\mathrm{NH}_{4}{ }^{+}\right)$, de l'orthophosphate $\left(\mathrm{PO}_{4}{ }^{-3}\right)$ et de la demande chimique et biochimique en oxygène ((DCO et $\left.\mathrm{DBO}_{5}\right)$ ont été déterminées conformément aux prescriptions de Rodier et al (2009) et afnor (2001). Les valeurs du rapport $\mathrm{DCO} / \mathrm{DBO}_{5}$ permettront d'identifier (Yapo et $a l, 2009$ ) :

-les effluents biodégradables ou effluents d'industrie agroalimentaire $\left(\mathrm{DCO} / \mathrm{DBO}_{5}<2\right)$

-les effluents biodégradables avec des souches sélectionnées ou effluents urbains domestiques $\left(2<\mathrm{DCO} / \mathrm{DBO}_{5}<3\right)$; 
-les effluents non biodégradables ou effluents plus ou moins biodégradables $\left(\mathrm{DCO} / \mathrm{DBO}_{5}>3\right.$.

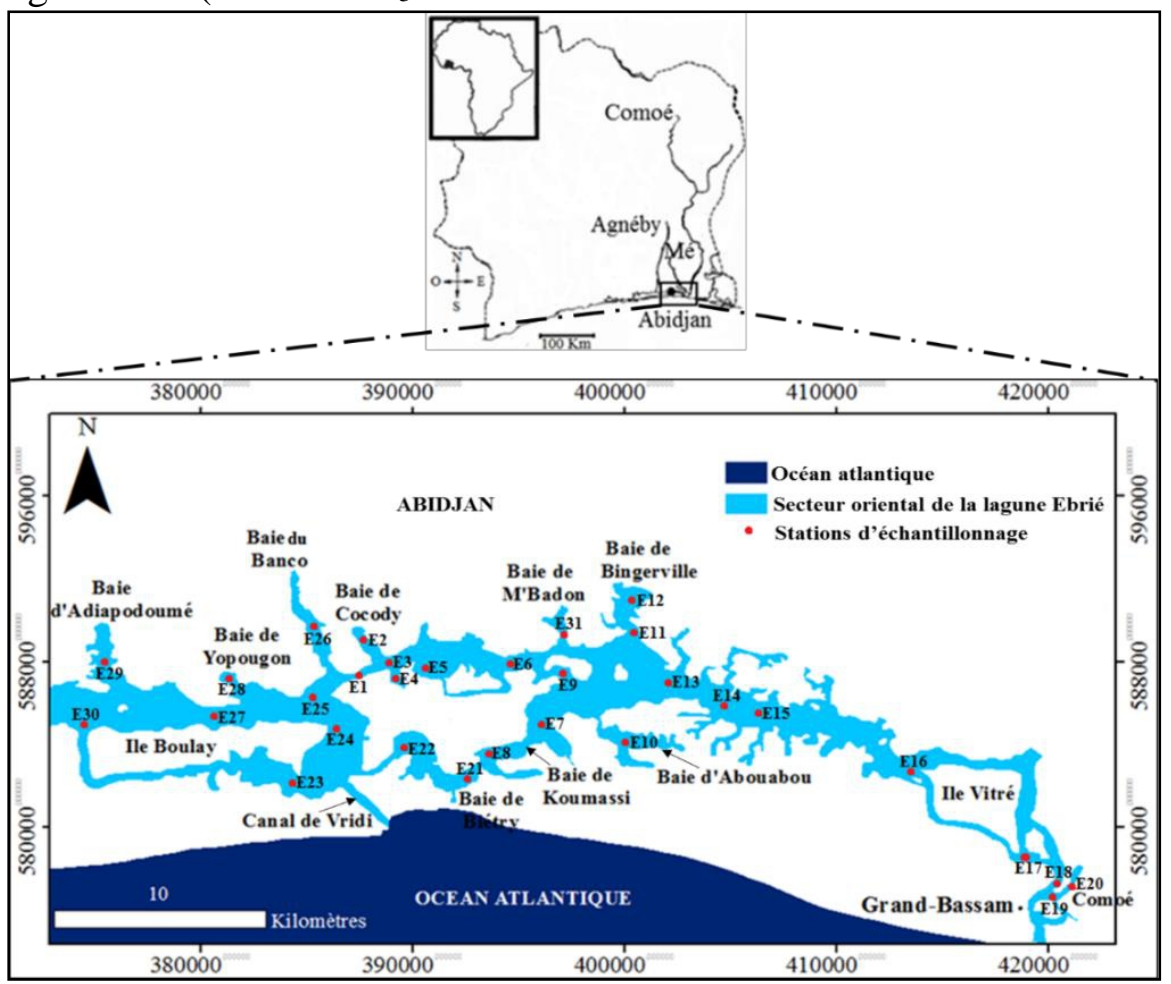

Figure 1 : Stations de mesure des paramètres physico-chimiques et de prélèvement des échantillons d'eau

\section{Traitement statistique des données}

Le traitement statistique a concerné toutes les données obtenues en période pluvieuse et en période sèche. La première approche a consisté à la détermination des corrélations entre les paramètres physico-chimiques étudiés. Une échelle des corrélations est utilisée pour l'interprétation des valeurs de corrélation (Figure 2). Dans la seconde approche, une analyse hiérarchique a permis de regrouper les observations ou stations et d'identifier les paramètres responsables de ce regroupement. La classification des stations d'échantillonnage est faite sur la base des teneurs de la demande chimique en oxygène $(\mathrm{DCO})$. Les autres paramètres permettront de dégager les spécificités de certains ensembles. L'outil utilisé est le logiciel Statisticat 7.1.

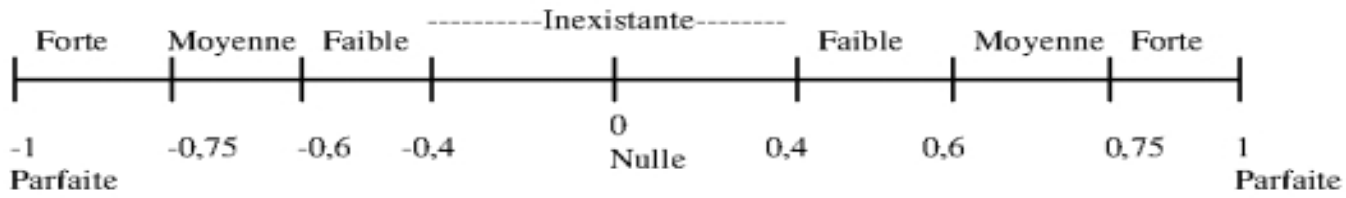

Figure 2 : Echelle des corrélations (Lacroix, 2006) 


\section{Resultats}

\section{Caractéristiques des paramètres étudiés}

Les tableaux I et II présentent les valeurs des paramètres physicochimiques mesurés sur le terrain et dosés au laboratoire pendant la saison pluvieuse (SP) et la saison sèche (SS).

Tableau I : Valeurs du pH, de la température, de la salinité, de l'oxygène et de la demande chimique en oxygène pendant deux saisons dans le secteur oriental de la lagune Ebrié

\begin{tabular}{|c|c|c|c|c|c|c|c|c|c|c|}
\hline \multirow{2}{*}{$\mathrm{N}^{\circ}$} & \multicolumn{2}{|c|}{$\mathrm{pH}$} & \multicolumn{2}{|c|}{$\mathrm{T}\left({ }^{\circ} \mathrm{C}\right)$} & \multicolumn{2}{|c|}{ Sal } & \multicolumn{2}{|c|}{$\mathrm{O}_{2}(\mathrm{mg} / \mathrm{L})$} & \multicolumn{2}{|c|}{$\mathrm{DCO}(\mathrm{mg} / \mathrm{L})$} \\
\hline & SP & SS & SP & SS & SP & SS & SP & SS & $\mathrm{SP}$ & SS \\
\hline E1 & 6,24 & 6,61 & 26,9 & 27 & 9,51 & 27,86 & 8,5 & 2,91 & 160 & 98 \\
\hline E2 & 5,22 & 7,33 & 26,4 & 27,88 & 7,6 & 25,86 & 2,28 & 2,38 & 75 & 50 \\
\hline E3 & 5,8 & 7,13 & 26,79 & 28,43 & 7,83 & 24,15 & 7,5 & 2,65 & 60 & 56 \\
\hline E4 & 6 & 7,03 & 25,98 & 28,33 & 11,75 & 25,27 & 7,65 & 2,06 & 82 & 56 \\
\hline E5 & 5,9 & 6,57 & 26,62 & 28,22 & 8,73 & 25,85 & 6,86 & 2,04 & 72 & 65 \\
\hline E6 & 5,93 & 6,49 & 27,99 & 28,82 & 2,36 & 23,27 & 7,68 & 2,83 & 77 & 77 \\
\hline E7 & 8,81 & 7,22 & 28,78 & 30,57 & 1,66 & 19,6 & 8,4 & 4,19 & 45 & 34 \\
\hline E8 & 6,62 & 7,56 & 29,45 & 30,19 & 4,22 & 19,5 & 9,55 & 3,31 & 29 & 25 \\
\hline E9 & 5,97 & 7,02 & 28,44 & 29,42 & 1,57 & 21,11 & 9,21 & 3 & 32 & 24 \\
\hline E10 & 5,79 & 7,58 & 28,87 & 30,3 & 1,52 & 16,47 & 6,51 & 3,67 & 63 & 69 \\
\hline E11 & 5,4 & 7,51 & 28,06 & 30,4 & 0,99 & 17,96 & 6,48 & 4,07 & 126 & 108 \\
\hline E12 & 5,84 & 7,41 & 28,34 & 29,95 & 0,75 & 17,67 & 5,57 & 4,02 & 93 & 72 \\
\hline E13 & 5,6 & 7,18 & 28,36 & 30,65 & 0,52 & 17,22 & 6,89 & 3,92 & 136 & 112 \\
\hline E14 & 5,66 & 7,36 & 28,4 & 30,62 & 0,44 & 15,93 & 5,7 & 4,15 & 13 & 11 \\
\hline E15 & 5,65 & 7,34 & 28,07 & 30,93 & 0,21 & 13,8 & 4,79 & 3,77 & 43 & 26 \\
\hline E16 & 5,3 & 7,2 & 28,14 & 31,39 & 0,37 & 11,61 & 5,51 & 3,82 & 49 & 40 \\
\hline E17 & 5,25 & 7,15 & 28,03 & 31,13 & 0,57 & 4,77 & 5,17 & 5,08 & 47 & 48 \\
\hline E18 & 5,7 & 7,16 & 27,92 & 30,8 & 0,16 & 3,12 & 3,33 & 5,69 & 40 & 17 \\
\hline E19 & 5,92 & 7,02 & 27,74 & 30,84 & 0,05 & 2,11 & 6,98 & 5,86 & 56 & 38 \\
\hline E20 & 6,62 & 6,97 & 27,75 & 30,44 & 0,05 & 1,4 & 6,84 & 6,56 & 119 & 70 \\
\hline E21 & 5,86 & 8,23 & 27,31 & 30,27 & 8,35 & 25,86 & 5,2 & 5,49 & 88 & 78 \\
\hline E22 & 6,11 & 7,73 & 27,03 & 29,21 & 9,77 & 28,36 & 5,73 & 3,97 & 96 & 84 \\
\hline E23 & 5,98 & 7,4 & 26,7 & 28,52 & 13,43 & 29,43 & 3,83 & 4,6 & 97 & 60 \\
\hline E24 & 5,74 & 7,45 & 26,39 & 27,49 & 10,06 & 27,51 & 6,63 & 3,02 & 98 & 64 \\
\hline E25 & 6,05 & 7,6 & 26,98 & 27,5 & 9,43 & 27,36 & 4,85 & 3,71 & 60 & 49 \\
\hline E26 & 5,57 & 7,45 & 27,09 & 28,81 & 9,92 & 24,85 & 3,35 & 3,62 & 72 & 58 \\
\hline E27 & 5,83 & 7,72 & 27,16 & 29,12 & 7,22 & 22,05 & 5,77 & 3,51 & 105 & 85 \\
\hline E28 & 5,76 & 7,55 & 27,24 & 28,47 & 9,57 & 24,68 & 3,86 & 3,18 & 69 & 49 \\
\hline E29 & 6,35 & 7,88 & 28,03 & 30,77 & 4,82 & 20,99 & 8,13 & 3,88 & 33 & 66 \\
\hline
\end{tabular}




\begin{tabular}{|c|c|c|c|c|c|c|c|c|c|c|}
\hline E30 & 6 & 7,13 & 27,3 & 30,63 & 5,41 & 18,59 & 6,5 & 2,95 & 93 & 56 \\
\hline E31 & 7 & 6,62 & 26,7 & 30,47 & 6,6 & 19,53 & 5,2 & 3,37 & 130 & 78 \\
\hline Max. & 8,81 & 8,23 & 29,45 & 31,39 & 13,43 & 29,43 & 9,55 & 6,56 & 160 & 112 \\
\hline Min. & 5,22 & 6,49 & 25,98 & 27 & 0,05 & 1,4 & 2,28 & 2,04 & 13 & 11 \\
\hline Moy. & 5,98 & 7,28 & 27,58 & 29,60 & 5,01 & 19,47 & 6,14 & 3,78 & 76,06 & 58,81 \\
\hline Ecart-type & 0,65 & 0,390 & 0,84 & 1,25 & 4,29 & 7,90 & 1,77 & 1,08 & 35,06 & 25,15 \\
\hline
\end{tabular}

Tableau II : Valeurs de la demande biochimique en oxygène, de l'orthophosphate, du nitrite, du nitrate et de l'ammonium pendant deux saisons dans le secteur oriental de la lagune Ebrié

\begin{tabular}{|c|c|c|c|c|c|c|c|c|c|c|}
\cline { 2 - 15 } \multicolumn{2}{c|}{} & \multicolumn{2}{|c|}{$\mathrm{DBO}_{5}(\mathrm{mg} / \mathrm{L})$} & \multicolumn{2}{|c|}{$\mathrm{PO}_{4}{ }^{3-}(\mathrm{mg} / \mathrm{L})$} & \multicolumn{2}{|c|}{$\mathrm{NO}_{3}{ }^{-}(\mathrm{mg} / \mathrm{L})$} & \multicolumn{2}{|c|}{$\mathrm{NO}_{2}{ }^{-}(\mathrm{mg} / \mathrm{L})$} & \multicolumn{2}{|c|}{$\mathrm{NH}_{4}{ }^{+}(\mathrm{mg} / \mathrm{L})$} \\
\hline $\mathrm{N}^{\circ}$ & $\mathrm{SP}$ & $\mathrm{SS}$ & $\mathrm{SP}$ & $\mathrm{SS}$ & $\mathrm{SP}$ & $\mathrm{SS}$ & $\mathrm{SP}$ & $\mathrm{SS}$ & $\mathrm{SP}$ & $\mathrm{SS}$ \\
\hline E1 & 70 & 56 & 0,3 & 1,13 & 26 & 0,3 & 0,165 & 0,021 & 0,2 & 0,141 \\
\hline E2 & 40 & 35 & 1,01 & 3,18 & 28 & 0,35 & 0,136 & 0,066 & 0,27 & 1,485 \\
\hline E3 & 35 & 32 & 0,35 & 6,45 & 23 & 0,13 & 0,107 & 0,062 & 0,19 & 1,646 \\
\hline E4 & 40 & 34 & 0,37 & 2,33 & 11 & 0,28 & 0,147 & 0,1 & 0,28 & 0,303 \\
\hline E5 & 41 & 37 & 0,99 & 1,3 & 35 & 0,09 & 0,127 & 0,062 & 0,04 & 0,236 \\
\hline E6 & 39 & 39 & 0,46 & 1,16 & 5,9 & 0,18 & 0,042 & 0,08 & 0,03 & 2,741 \\
\hline E7 & 20 & 18 & 0,58 & 1,75 & 0,1 & 0,13 & 0,028 & 0,005 & 0,03 & 0,028 \\
\hline E8 & 17 & 14 & 2 & 4,61 & 57 & 0,15 & 0,288 & 0,068 & 0,05 & 0,434 \\
\hline E9 & 20 & 15 & 0,66 & 0,87 & 2,5 & 0,17 & 0,023 & 0,1 & 0,04 & 0,041 \\
\hline E10 & 40 & 43 & 0,43 & 6,62 & 0,05 & 0,01 & 0,007 & 0,007 & 0,04 & 0,022 \\
\hline E11 & 82 & 68 & 0,95 & 1,17 & 0,01 & 0,08 & 0,008 & 0,002 & 0,04 & 0,119 \\
\hline E12 & 40 & 38 & 0,23 & 1,15 & 0,08 & 0,18 & 0,008 & 0,005 & 0,05 & 0,058 \\
\hline E13 & 70 & 58 & 0,1 & 0,72 & 3,2 & 0,09 & 0,007 & 0,003 & 0,05 & 0,099 \\
\hline E14 & 5 & 6 & 3,4 & 0,92 & 0,19 & 0,009 & 0,015 & 0,002 & 0,11 & 0,038 \\
\hline E15 & 30 & 15 & 1,93 & 0,92 & 5,3 & 0,11 & 0,012 & 0,004 & 0,13 & 0,055 \\
\hline E16 & 36 & 26 & 0,77 & 0,82 & 0,015 & 0,09 & 0,001 & 0,002 & 0,05 & 0,07 \\
\hline E17 & 22 & 26 & 0,1 & 0,59 & 0,17 & 0,11 & 0,021 & 0,004 & 0,08 & 0,074 \\
\hline E18 & 20 & 12 & 3,2 & 0,95 & 3,9 & 0,12 & 0,008 & 0,018 & 0,03 & 0,078 \\
\hline E19 & 25 & 20 & 0,97 & 1,94 & 2,3 & 0,01 & 0,007 & 0,008 & 0,04 & 0,12 \\
\hline E20 & 60 & 46 & 0,02 & 1,21 & 1,3 & 0,01 & 0,007 & 0,033 & 0,05 & 0,082 \\
\hline E21 & 60 & 42 & 1,5 & 5,01 & 0,97 & 0,7 & 0,144 & 0,018 & 0,75 & 0,235 \\
\hline E22 & 56 & 48 & 5,3 & 1,46 & 1,1 & 0,3 & 0,137 & 0,009 & 1 & 0,047 \\
\hline E23 & 53 & 48 & 2,9 & 0,51 & 18,5 & 0,2 & 0,234 & 0,009 & 0,01 & 0,041 \\
\hline E24 & 60 & 39 & 0,67 & 1,66 & 1,2 & 0,3 & 0,015 & 0,015 & 0,1 & 0,065 \\
\hline E25 & 26 & 25 & 1,4 & 0,59 & 7,3 & 0,2 & 0,133 & 0,017 & 0,03 & 0,23 \\
\hline E26 & 50 & 30 & 3,8 & 1,53 & 2,4 & 0,4 & 0,175 & 0,026 & 0,75 & 0,248 \\
\hline
\end{tabular}




\begin{tabular}{|c|c|c|c|c|c|c|c|c|c|c|}
\hline E27 & 61 & 50 & 0,17 & 1,12 & 2,4 & 0,3 & 0,134 & 0,06 & 0,01 & 0,46 \\
\hline E28 & 30 & 26 & 3,9 & 1,163 & 1,2 & 1,3 & 0,163 & 0,117 & 0,41 & 2,23 \\
\hline E29 & 16 & 38 & 4,5 & 1,01 & 10,6 & 0,3 & 0,126 & 0,04 & 0,19 & 0,124 \\
\hline E30 & 58 & 29 & 0,11 & 0,93 & 2,5 & 0,2 & 0,154 & 0,036 & 0,03 & 0,053 \\
\hline E31 & 25 & 46 & 5,6 & 0,41 & 0,4 & 0,21 & 0,18 & 0,126 & 2,5 & 0,107 \\
\hline Max. & 82 & 68 & 5,6 & 6,62 & 57 & 1,3 & 0,288 & 0,126 & 2,5 & 2,741 \\
\hline Min. & 5 & 6 & 0,02 & 0,41 & 0,01 & 0,009 & 0,001 & 0,002 & 0,01 & 0,022 \\
\hline Moy. & 40,22 & 34,16 & 1,57 & 1,78 & 8,18 & 0,226 & 0,089 & 0,036 & 0,244 & 0,378 \\
\hline Ecart-type & 18,97 & 14,77 & 1,65 & 1,65 & 13,09 & 0,24 & 0,08 & 0,04 & 0,48 & 0,68 \\
\hline
\end{tabular}

\section{Variation des paramètres analysés sur le terrain $\left(\mathrm{pH}, \mathrm{T}^{\circ}\right.$, Sal et $\left.\mathrm{O}_{2}\right)$}

La valeur maximale du $\mathrm{pH}$ des eaux de la partie orientale de la lagune Ebrié en saison pluvieuse est de 8,81. Elle a été mesurée dans la baie de Koumassi (E7). Quant à la valeur minimale qui est de 5,22, elle a été enregistrée dans la baie de Cocody (E2). En saison sèche, le $\mathrm{pH}$ maximal est égal à 8,23. Les moyennes respectives du $\mathrm{pH}$ des eaux, pendant la saison des pluies et la saison sèche, sont de 5,98 et de 7,28. Les eaux sont acides en période pluvieuse et alcalines en période sèche.

La variation saisonnière de la température se fait entre $25,98{ }^{\circ} \mathrm{C}$ et $31,39^{\circ} \mathrm{C}$. La température la plus forte a été mesurée pendant la saison sèche entre la baie de Bingerville et l'Île Vitré (E16). La plus faible valeur de température a été enregistrée pendant la saison pluvieuse à la station M'Pouto (E6). Les températures moyennes en saison des pluies et en saison sèche sont respectivement 27,58 et $29,6{ }^{\circ} \mathrm{C}$. Les eaux du secteur oriental de la lagune Ebrié sont plus chaudes en saison sèche.

La salinité oscille de 0,05 à 13,4 en la saison pluvieuse. Ces valeurs sont obtenues aux stations E19 et E20 pour la salinité minimale et à la station E29 pour la salinité maximale. En absence de pluies, les valeurs extrêmes de la salinité sont 1,4 (E20) et 29,43 (E23). Elles ont été mesurées respectivement dans le fleuve Comoé et en face du canal de Vridi. La salinité moyenne est de 5,01 en saison des pluies et de 19,47 en saison sèche. Les eaux lagunaires ont une salinité forte dans le mois de février (saison sèche).

Les teneurs de l'oxygène dissous se trouvent entre $2,28 \mathrm{mg} / \mathrm{L}$ (E2) et 9,55 mg/L (E8) en saison pluvieuse et entre 2,04 mg/L (E6) et $6,56 \mathrm{mg} / \mathrm{L}$ (E20) en saison sèche. Les teneurs moyennes respectives sont $6,14 \mathrm{mg} / \mathrm{L}$ et 3,78 $\mathrm{mg} / \mathrm{L}$. Les valeurs de l'oxygène sont plus fortes en saison des pluies. 


\section{Variation saisonnière des teneurs en nutriments \\ L'ammonium $\left(\mathrm{NH}_{4}{ }^{+}\right)$}

En période pluvieuse, les teneurs en $\mathrm{NH}_{4}{ }^{+}$se situent entre 0,01 et 2,5 $\mathrm{mg} / \mathrm{L}$. La teneur moyenne est de $0,244 \mathrm{mg} / \mathrm{L}$. Les fortes concentrations s'observent dans les baies de Cocody (E2), de Marcory (E4), de Biétry (E21 et E22), du Banco (E26), de Yopougon (E28) et de M'Badon (E31). La plus faible teneur $(0,01 \mathrm{mg} / \mathrm{L})$ est enregistrée dans les eaux en face de la centrale d'Azito (E27) et à l'entrée du canal de Vridi (E23). Les teneurs en ammonium pendant la période sèche se trouvent entre 0,022 et $2,741 \mathrm{mg} / \mathrm{L}$. La moyenne des teneurs est de $0,378 \mathrm{mg} / \mathrm{L}$. Elle est supérieure à celle de la période pluvieuse. Les eaux de la baie d'Abouabou ont la teneur en ammonium la plus basse en février. Les baies de Cocody (E2 et E3), de Koumassi (E8), de Biétry (E21), du Banco (E26), de Yopougon (E28) et de M'Badon (E31) ont de fortes concentrations en ammonium dans leurs eaux.

\section{Le nitrite $\left(\mathrm{NO}_{2}{ }^{-}\right)$}

Les teneurs sont comprises entre 0,001 et $0,288 \mathrm{mg} / \mathrm{L}$ en saison pluvieuse. La moyenne est égale à $0,089 \mathrm{mg} / \mathrm{L}$. Les valeurs obtenues sont élevées dans les baies d'Adiapodoumé, de Cocody et de Marcory, au sud de la baie de Koumassi (E8) et en face du canal de Vridi (E23) et du palais de la culture (E1). Elles sont faibles dans les baies de Bingerville (E11 et E12), d'Abouabou et de M'Badon, à l'Est de la baie de Biétry, au nord de la baie de Koumassi (E7) et dans le chenal au Sud de l'Île Vitré (E16 et E17). Les concentrations en nitrite pendant la période sèche oscillent entre 0,002 et 0,126 $\mathrm{mg} / \mathrm{L}$. La valeur moyenne est de $0,036 \mathrm{mg} / \mathrm{L}$. Cette teneur moyenne est inférieure à celle de la saison pluvieuse. Les eaux des baies de Cocody (E2), de Marcory (E4), de Biétry (E21 et E22), du Banco (E26), de Yopougon (E28) et d'Adiapodoumé (E29) sont fortement concentrées en nitrite. Les eaux ayant les plus faibles concentrations en nitrite se rencontrent à l'entrée de la baie de Bingerville (E11), dans la baie d'Abouabou et dans le fleuve Comoé (E20).

\section{Le nitrate $\left(\mathrm{NO}_{3}{ }^{-}\right)$}

Dans le secteur oriental de la lagune Ebrié, les teneurs en nitrate oscillent en période pluvieuse entre 0,01 et $57 \mathrm{mg} / \mathrm{L}$. La concentration moyenne est de de $8,18 \mathrm{mg} / \mathrm{L}$. Les teneurs sont faibles $(<1 \mathrm{mg} / \mathrm{L})$ dans le chenal au Sud de l'île Vitré et dans les baies de Bingerville, Abouabou et de M'Badon. De l'île Vitré au fleuve Comoé, les teneurs sont comprises entre 1 et $4 \mathrm{mg} / \mathrm{L}$. Dans les baies de Koumassi, d'Adiapodoumé, de Cocody, de Marcory et du Banco les concentrations enregistrées sont plus fortes. La période sèche est marquée par une teneur minimale de $0,009 \mathrm{mg} / \mathrm{L}$ et une teneur maximale de $1,3 \mathrm{mg} / \mathrm{L}$. Ces valeurs extrêmes s'observent respectivement juste après la baie d'Anna (E14) et dans la baie de Yopougon 
(E28). La teneur moyenne est de $0,23 \mathrm{mg} / \mathrm{L}$. Les teneurs en nitrate pendant la période sèche sont presque toutes faibles $(<1 \mathrm{mg} / \mathrm{L})$, sauf dans la baie de Yopougon où la teneur en nitrate est maximale. La teneur moyenne en saison pluvieuse $(8,18 \mathrm{mg} / \mathrm{L})$ est supérieure à la teneur moyenne en saison sèche $(0,23$ $\mathrm{mg} / \mathrm{L})$.

\section{Les orthophosphates $\left(\mathrm{PO}_{4}{ }^{3-}\right)$}

La concentration la plus forte en orthophosphate en période pluvieuse du secteur oriental de la lagune Ebrié est enregistrée dans la baie de M'Badon $(5,6 \mathrm{mg} / \mathrm{L})$. La concentration la plus faible quant à elle est obtenue dans les eaux du fleuve Comoé $(0,02 \mathrm{mg} / \mathrm{L})$. Les teneurs sont élevées au niveau des eaux de la zone d'Abidjan. En période sèche, la baie d'Abouabou a la teneur la plus forte $(6,62 \mathrm{mg} / \mathrm{L})$ et la baie de M'Badon, la teneur la plus faible $(0,41$ $\mathrm{mg} / \mathrm{L}$ ) (Figure 3). Les teneurs moyennes en période sèche et pluvieuse sont respectivement $1,78 \mathrm{mg} / \mathrm{L}$ et $1,57 \mathrm{mg} / \mathrm{L}$. La teneur moyenne en orthophosphate en période pluvieuse est inférieure à celle de la période sèche.

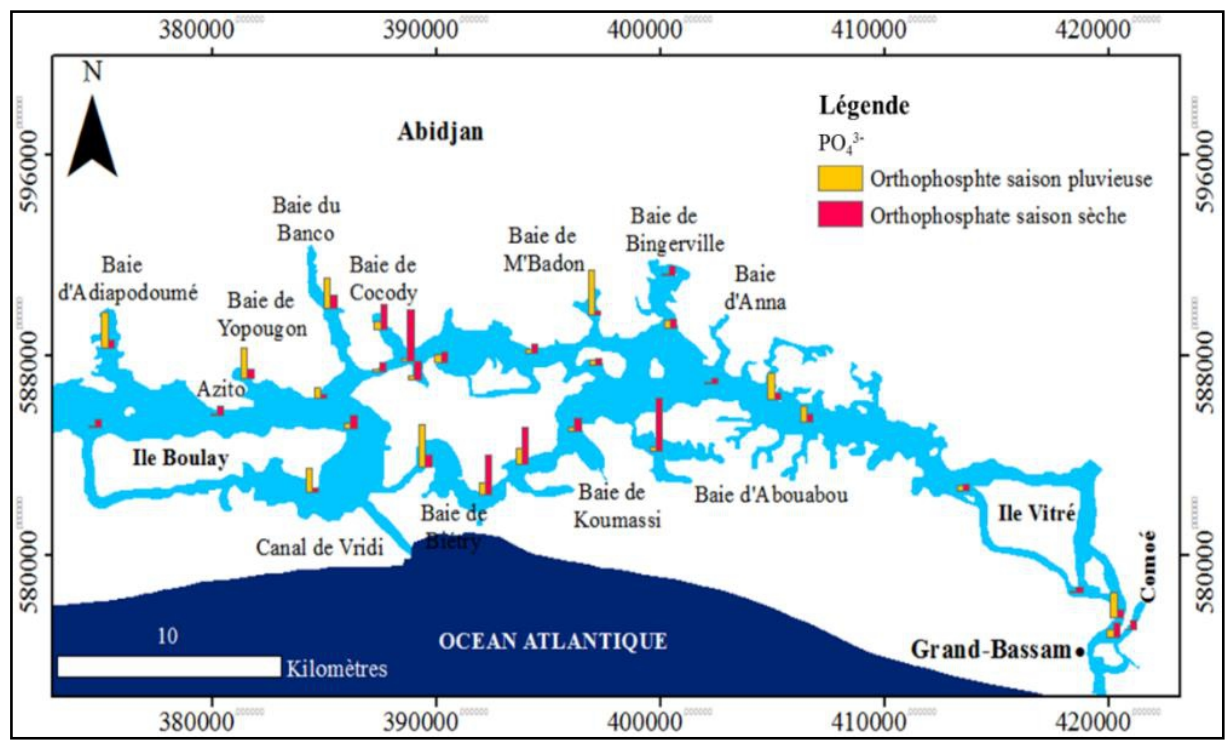

Figure 3 : Distribution spatiale des teneurs des orthophosphates en saison pluvieuse et sèche

Evolution des teneurs de la demande chimique et biochimique en oxygène La Demande Chimique en Oxygène (DCO)

La demande chimique en oxygène (DCO) est la quantité d'oxygène consommée lors de la décomposition chimique des composés organiques et des sels minéraux oxydables dans l'eau. Les valeurs de la DCO en période pluvieuse varient de 13 à $160 \mathrm{mg} / \mathrm{L}$, avec une moyenne de 76,06 mg/L. La station (E1) en face du palais de la culture d'Abidjan enregistre la plus forte valeur. La plus faible teneur est obtenue à la station située après la baie d'Anna 
(E14). La période sèche est marquée par des valeurs qui oscillent entre 11 et $112 \mathrm{mg} / \mathrm{L}$. La plus forte valeur est obtenue en face de la baie d'Anna (E13). La valeur la plus faible est enregistrée au niveau de la station (E14) située juste après la baie d'Anna (Figure 4). La teneur moyenne de la DCO est de 58,81 $\mathrm{mg} / \mathrm{L}$ en période sèche. Cette valeur est inférieure à celle de la période pluvieuse.

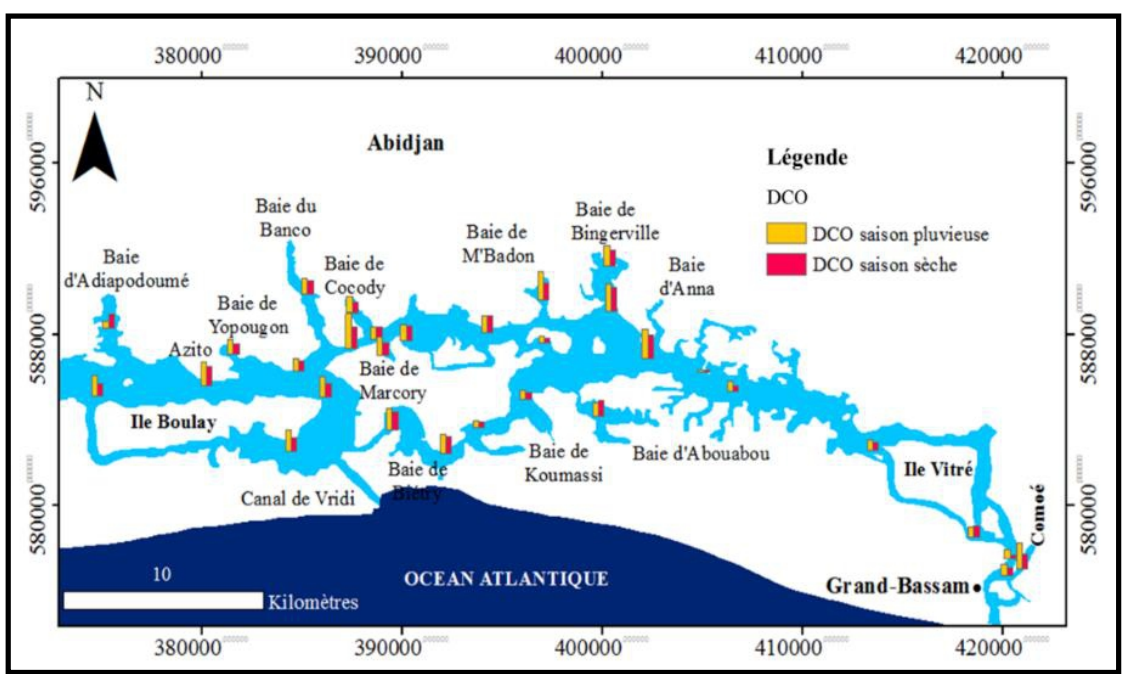

Figure 4 : Variation spatiale des valeurs de la DCO dans la partie Est de la lagune Ebrié

\section{La Demande Biochimique en Oxygène (DBO5)}

La demande biochimique en oxygène $\left(\mathrm{DBO}_{5}\right)$ représente la quantité d'oxygène nécessaire aux bactéries pour dégrader l'ensemble de la matière organique contenue dans un échantillon d'eau à $20^{\circ} \mathrm{C}$, pendant 5 jours à l'obscurité. Les teneurs de la $\mathrm{DBO}_{5}$ évoluent sur l'ensemble de la zone entre 5 et $82 \mathrm{mg} / \mathrm{L}$ en période pluvieuse. Par contre, en saison sèche, les teneurs varient de 6 à $68 \mathrm{mg} / \mathrm{L}$ (Figure 5). La baie de Bingerville enregistre la valeur de la $\mathrm{DBO}_{5}$ la plus forte en saison pluvieuse et en saison sèche. Ce constat serait lié au faible renouvellement des eaux et aux activités anthropiques. Les valeurs de la $\mathrm{DBO}_{5}$ sont faibles avant l'île Vitré et dans la baie de Koumassi. La teneur moyenne en période sèche $(34,16 \mathrm{mg} / \mathrm{L})$ est inférieure à celle de la période pluvieuse $(40,23 \mathrm{mg} / \mathrm{L})$. 


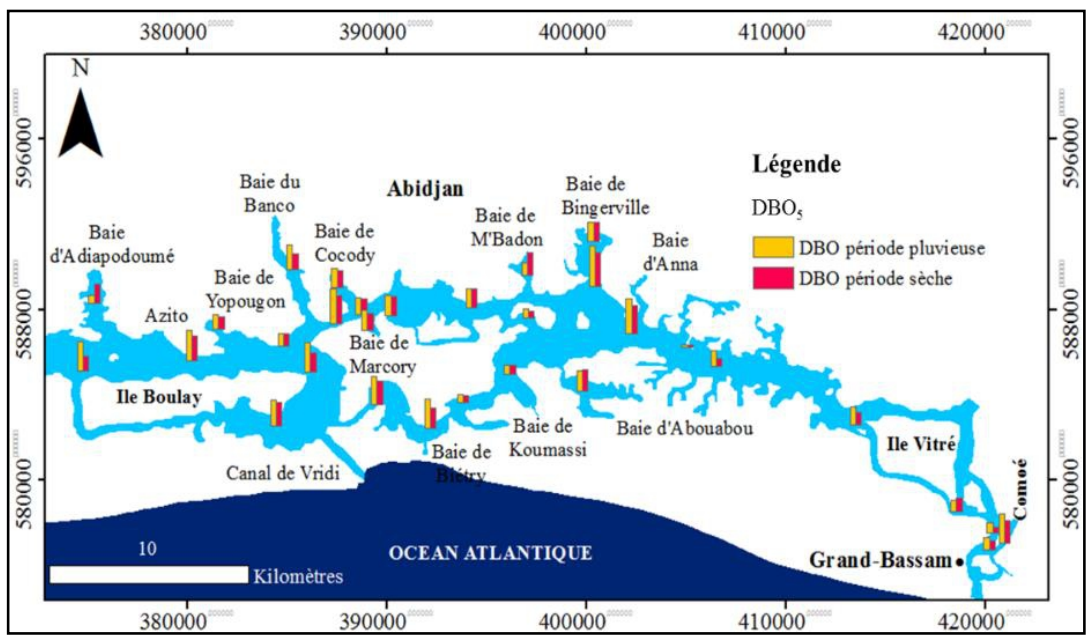

Figure 5 : Répartition spatio-temporelle des valeurs de la $\mathrm{DBO}_{5}$

\section{Rapport DCO/DBO5}

Le ratio $\mathrm{DCO} / \mathrm{DBO}_{5}$ évolue entre 1,36 et 5,2 pendant la saison pluvieuse. Les eaux ayant un ratio inférieur à 2 contiennent des substances biodégradables. Elles sont le plus représentées au niveau de la région orientale de la lagune Ebrié. Les échantillons d'eau dont le ratio se situe entre 2 et 3 contiennent des substances biodégradables avec des souches sélectionnées. Ils se rencontrent dans les baies de Marcory, Koumassi, Yopougon, Adiapodoumé, Bingerville et dans les eaux au niveau de Grand-Bassam. Les eaux de la baie de M'Badon indiquent un ratio supérieur à 3 (Figure 6). Elles contiennent des substances non biodégradables ou plus ou moins difficilement biodégradables. En période sèche, les rapports $\mathrm{DCO} / \mathrm{DBO}_{5}$ évoluent entre 1,25 et 1,97 ; ce qui implique que les eaux lagunaires contiennent essentiellement des matières biodégradables provenant principalement des activités agroindustrielles et domestiques. La prédominance des activités agro-alimentaires se remarque bien, avec la répartition des substances biodégradables dans presque tout le secteur oriental de la lagune Ebrié. 


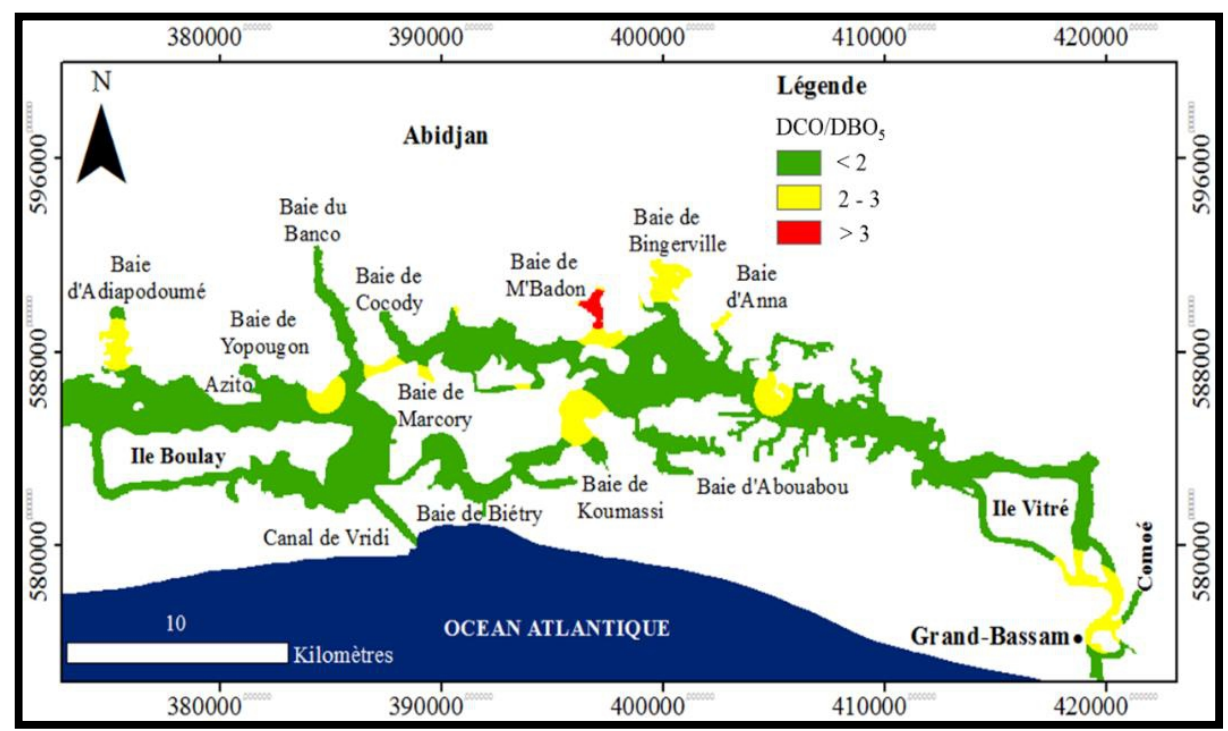

Figure 6 : Caractéristiques des eaux du secteur oriental en fonction du rapport $\mathrm{DCO} / \mathrm{DBO}_{5}$ pendant la saison pluvieuse

\section{Corrélations entre les paramètres étudiés}

La matrice de corrélations entre les paramètres des eaux en saison pluvieuse (Tableau III) permet de voir une bonne corrélation positive entre la DCO et la $\mathrm{DBO}_{5}\left(\mathrm{r}=0,84\right.$ et entre $\mathrm{NO}_{2}{ }^{-}$et $\mathrm{Sal}(\mathrm{r}=0,76)$. La corrélation entre la $\mathrm{DCO}$ et le $\mathrm{pH}$ est nulle. On observe une bonne corrélation négative entre $\mathrm{T}^{\circ}$ et $\mathrm{Sal}(\mathrm{r}=-0,79)$. Pendant la saison sèche, les bonnes corrélations positives

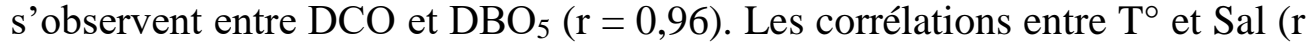
$=-0,75)$ bonnes et négatives. Le $\mathrm{pH}$ et la $\mathrm{DCO}$ ont une corrélation nulle (Tableau IV).

Tableau III : Matrice de corrélation entre les paramètres physiques et chimiques des eaux du secteur oriental en saison pluvieuse

\begin{tabular}{|c|c|c|c|c|c|c|c|c|c|c|}
\hline & DCO & $\mathrm{DBO}_{5}$ & $\mathrm{PO}_{4}^{3-}$ & $\mathrm{NO}_{3}{ }^{-}$ & $\mathrm{NO}_{2}^{-}$ & $\mathrm{NH}_{4}^{+}$ & $\mathrm{pH}$ & $\mathrm{T}^{\circ}$ & Sal & $\mathrm{O}_{2}$ \\
\hline DCO & 1 & 0,84 & $-0,15$ & $-0,09$ & 0,14 & 0,30 & 0,00 & $-0,43$ & 0,30 & $-0,03$ \\
\hline $\mathrm{DBO}$ & & 1 & $-0,31$ & $-0,10$ & 0,05 & $-0,03$ & $-0,22$ & $-0,34$ & 0,28 & $-0,04$ \\
\hline $\mathrm{PO}_{4}{ }^{3-}$ & & & 1 & $-0,05$ & 0,39 & 0,65 & 0,10 & $-0,12$ & 0,27 & $-0,35$ \\
\hline $\mathrm{NO}_{3}^{-}$ & & & & 1 & 0,61 & $-0,15$ & 0,05 & $-0,07$ & 0,32 & 0,26 \\
\hline $\mathrm{NO}_{2}^{-}$ & & & & & 1 & 0,35 & 0,15 & $-0,40$ & 0,76 & $-0,05$ \\
\hline $\mathrm{NH}_{4}{ }^{+}$ & & & & & & 1 & 0,20 & $-0,33$ & 0,29 & $-0,23$ \\
\hline $\mathrm{pH}$ & & & & & & & 1 & 0,20 & $-0,01$ & 0,45 \\
\hline $\mathrm{T}^{\circ}$ & & & & & & & & 1 & $-0,79$ & 0,36 \\
\hline Sal & & & & & & & & & 1 & $-0,19$ \\
\hline $\mathrm{O}_{2}$ & & & & & & & & & & 1 \\
\hline
\end{tabular}


Tableau IV : Matrice de corrélation entre les paramètres physiques et chimiques des eaux du secteur oriental en saison sèche

\begin{tabular}{|c|c|c|c|c|c|c|c|c|c|c|}
\hline & $\mathrm{DCO}$ & $\mathrm{DBO}_{5}$ & $\mathrm{PO}_{4}{ }^{3-}$ & $\mathrm{NO}_{3}^{-}$ & $\mathrm{NO}_{2}$ & $\mathrm{NH}_{4}^{+}$ & $\mathrm{pH}$ & $\mathrm{T}^{\circ}$ & Sal & $\mathrm{O}_{2}$ \\
\hline DCO & 1 & 0,96 & $-0,02$ & 0,09 & $-0,04$ & 0,04 & $\mathbf{0 , 0 0}$ & $-0,22$ & 0,32 & $-0,10$ \\
\hline $\mathrm{DBO}$ & & 1 & $-0,01$ & 0,05 & $-0,05$ & $-0,01$ & 0,01 & $-0,24$ & 0,31 & $-0,06$ \\
\hline $\mathrm{PO}_{4}{ }^{3-}$ & & & 1 & 0,03 & 0,05 & 0,21 & 0,29 & $-0,07$ & 0,14 & $-0,11$ \\
\hline $\mathrm{NO}_{3}{ }^{-}$ & & & & 1 & 0,42 & 0,47 & 0,36 & $-0,35$ & 0,44 & $-0,17$ \\
\hline $\mathrm{NO}_{2}^{-}$ & & & & & 1 & 0,53 & $-0,32$ & $-0,32$ & 0,29 & $-0,52$ \\
\hline $\mathrm{NH}_{4}{ }^{+}$ & & & & & & 1 & $-0,18$ & $-0,38$ & 0,27 & $-0,37$ \\
\hline $\mathrm{pH}$ & & & & & & & 1 & 0,13 & 0,18 & 0,25 \\
\hline $\mathrm{T}^{\circ}$ & & & & & & & & 1 & $-0,75$ & 0,58 \\
\hline Sal & & & & & & & & & 1 & $-0,69$ \\
\hline $\mathrm{O}_{2}$ & & & & & & & & & & 1 \\
\hline
\end{tabular}

\section{Analyse en Classification Hiérarchique Ascendante (CAH)}

Le dendrogramme de la figure 7 permet de distinguer deux (2) ensembles en période pluvieuse. Les stations dont les teneurs de la DCO sont comprises entre 88 et $160 \mathrm{mg} / \mathrm{L}$, constituent le premier ensemble ou ensemble (A). L'ensemble (B) concerne les stations ayant des teneurs de la DCO oscillant de 13 à $82 \mathrm{mg} / \mathrm{L}$. La figure 8 montre deux (2) ensembles en période sèche. L'ensemble $(\mathrm{O})$ regroupe les stations dont la teneur de la DCO est supérieure ou égale à $64 \mathrm{mg} / \mathrm{L}(\geq 64 \mathrm{mg} / \mathrm{L})$. Les autres stations formant l'ensemble $(\mathrm{P})$ ont une DCO inférieure à $64 \mathrm{mg} / \mathrm{L}$.

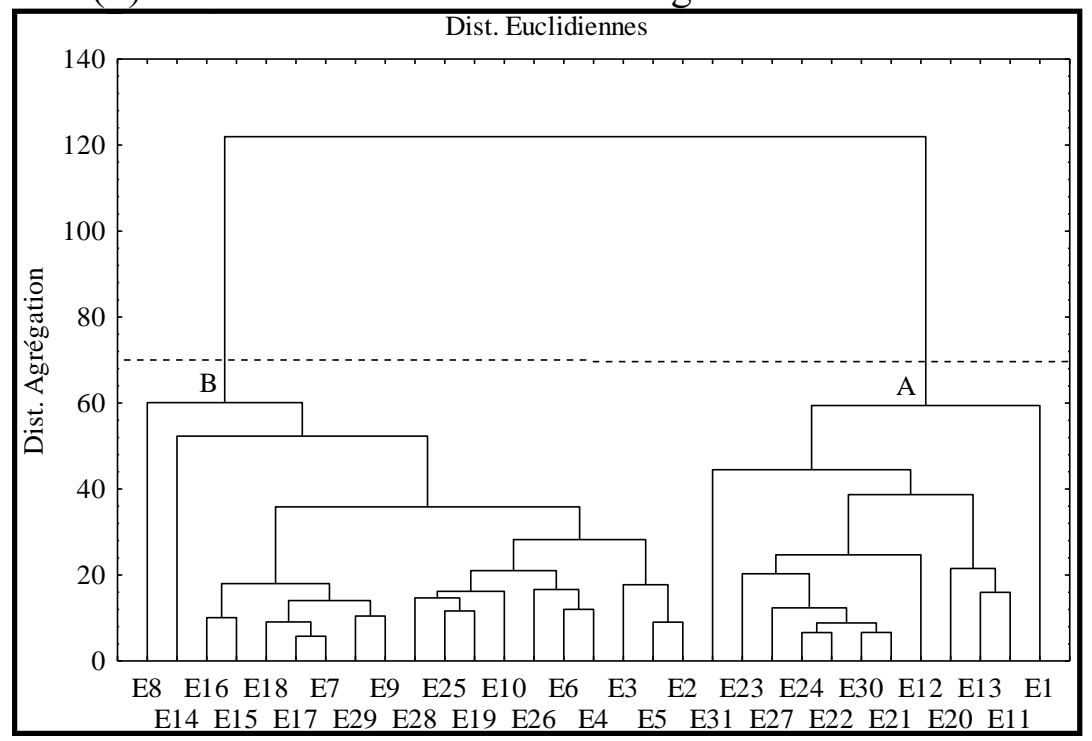

Figure 7 : Classification des stations d'échantillonnage en saison pluvieuse 


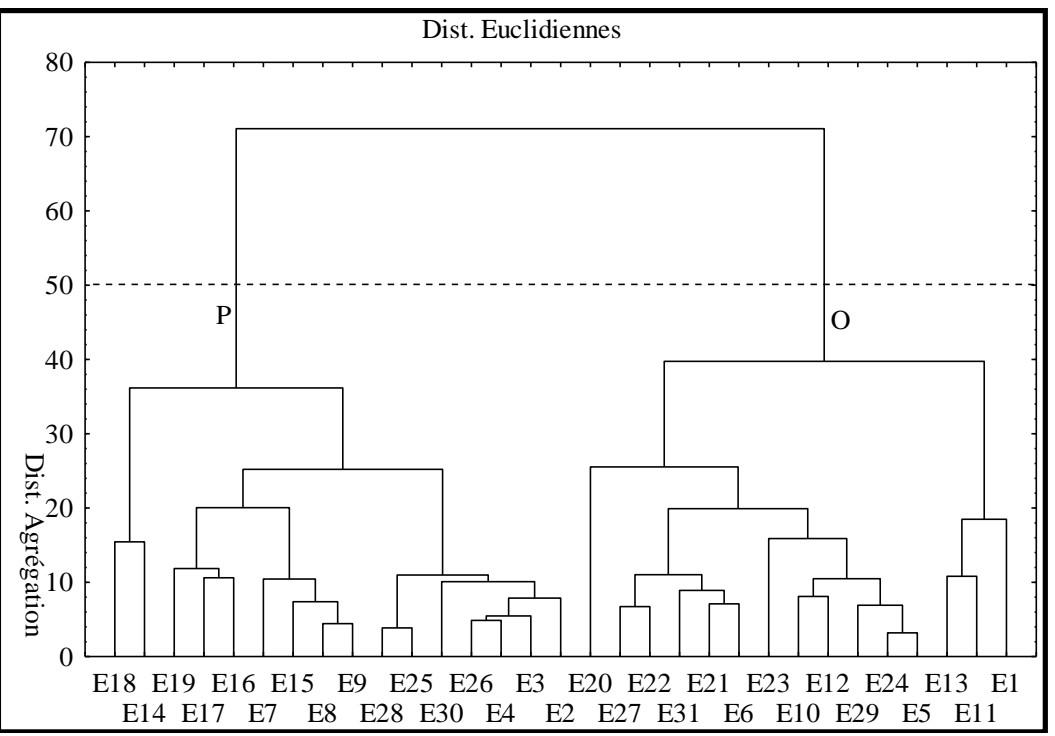

Figure 8 : Analyse Hiérarchique des stations d'échantillonnage en saison sèche

\section{Interpretations et discussion}

\section{$\checkmark \quad$ Variation des paramètres hydrologiques}

Les eaux de la lagune Ebrié sont acides $(\mathrm{pH}$ moyen $=5,98)$ en saison pluvieuse et alcalines $(\mathrm{pH}$ moyen $=7,28$ ) en saison sèche. Pendant la saison pluvieuse, les eaux continentales prédominent en lagune Ebrié à cause de la crue des rivières côtières (Agnéby, Banco, Mé). En saison sèche, les eaux marines sont plus abondantes. Selon Tastet (1974) et Dufour (1982), les eaux d'origine marine sont basiques $(\mathrm{pH}>7)$ et celles d'origine continentale sont acides $(\mathrm{pH}<7)$. Les variations du $\mathrm{pH}$, dans la lagune Ebrié, sont fonction de l'importance relative des eaux de l'une ou l'autre origine. La variation du $\mathrm{pH}$ dans la lagune Ebrié est identique à celle de la lagune de Grand-Lahou. Dans cette lagune, les eaux sont également acides en saison pluvieuse avec un $\mathrm{pH}$ moyen égal à 6,6 et basique en saison sèche avec un $\mathrm{pH}$ moyen égal à 8,46 (Konan et al, 2013).

Les valeurs extrêmes de la température enregistrées pendant les deux saisons sont $25,98^{\circ} \mathrm{C}$ et $31,39^{\circ} \mathrm{C}$. La variation de la température est conforme à celle trouvée par Pages et al (1979) et Dufour (1982) qui indiquent respectivement une variation saisonnière de la température entre $25,5^{\circ} \mathrm{C}$ et 34 ${ }^{\circ} \mathrm{C}$ et entre $22,5^{\circ} \mathrm{C}$ et $35^{\circ} \mathrm{C}$. Selon Dufour, (1982), les milieux frontières sont à l'origine de la variation spatiale de la température des eaux.

\section{Evolution des teneurs en sels nutritifs}

Les sels nutritifs dans les eaux lagunaires peuvent être divisés en deux groupes en fonction de leur concentration. Le premier groupe est celui du nitrite. Ses teneurs sont majoritairement faibles dans les eaux lagunaires par 
rapport aux autres nutriments. Les moyennes sont de $0,089 \mathrm{mg} / \mathrm{L}$ pour la saison des pluies et de $0,036 \mathrm{mg} / \mathrm{L}$ pour la saison sèche. Les faibles teneurs en nitrite pourraient être attribuées aux valeurs du $\mathrm{pH}$ et de l'oxygène dissous. En effet, les valeurs de ces deux paramètres influencent la nitrification et l'optimum est atteint pour des valeurs de $\mathrm{pH}$ situées entre 7 et 8 et des teneurs en oxygène dissous variant entre 5 et $10 \mathrm{mg} / \mathrm{L}$ (Shaher et $\mathrm{al}, 1993$ ). La teneur moyenne du nitrite en période sèche est inférieure à celle de la période pluvieuse. Cela pourrait s'expliquer par un faible apport en nitrite pendant la période sèche. La faible teneur moyenne en période sèche peut être due aussi à la dégradation de la matière organique présente dans le milieu lagunaire en période pluvieuse par rapport à la période sèche. Cette action transforme la matière organique en ammonium. L'ammonium est transformé en nitrite sous l'action des bactéries nitrifiantes, appelées Nitrosomas. Le deuxième groupe est celui de l'ammonium, de l'orthophosphate et du nitrate. Ils sont plus concentrés dans les eaux lagunaires par rapport au nitrite. Cette forte concentrations relative s'expliquerait par l'utilisation des engrais et fertilisant dans les différentes activités agricoles. A cela s'ajoute la dégradation de la matière organique sédimentée par les micro-organismes et les bactéries (Bamba et al, 2008). Les nutriments issus des activités agricoles atteignent le milieu aquatique grâce aux eaux de drainage des terrains agricoles des bassins versants (Hammami et $a l, 2005)$. Les nutriments des eaux de la lagune Ebrié ont deux origines principales: la contribution naturelle (apport d'eau continentale, apport océanique lié aux résurgences côtières, remise en suspension des sédiments organiques) et la contribution anthropique (effluents domestiques et industrielles) (Kouassi et al, 2005).La teneur moyenne de l'ammonium pendant la période pluvieuse $(0,24 \mathrm{mg} / \mathrm{L})$ est inférieure à celle de la période sèche $(0,378 \mathrm{mg} / \mathrm{L})$. Cette tendance traduirait l'effet de la bonne oxygénation des eaux en période pluvieuse entraînant l'oxydation de l'azote (Makhoukh et al, 2011). Concernant la teneur moyenne en orthophosphate, elle est supérieure pendant la période sèche par rapport à celle de la période pluvieuse. Cette diminution de la teneur moyenne en période pluvieuse peut s'expliquer par l'assimilation des orthophosphates par les bactéries et les algues (Hernandez et $a l, 1996$ ) ou à la dilution des teneurs en orthophosphate lors de la période pluvieuse. Par contre la forte teneur moyenne en période sèche pourrait être due à la proximité des sources d'orthophosphates (Irié, 2017).

\section{Variabilité saisonnière de la $\mathrm{DCO}$ et de la $\mathrm{DBO}_{5}$}

Les valeurs de la $\mathrm{DCO}$ et de la $\mathrm{DBO}_{5}$ sont plus fortes en saison pluvieuse qu'en saison sèche. Cela s'expliquerait par un apport important de matières organiques et minérales biodégradables dans la lagune Ebrié, pendant la saison pluvieuse, dont la décomposition est favorisée par une bonne oxygénation des eaux lagunaires (Irie, 2017). La forte teneur de la DCO à la 
station Palais de la culture (E1) serait due au rétrécissement du chenal lagunaire, à une insuffisance au niveau du renouvellement des eaux et aux activités humaines. Le rétrécissement du chenal entraine un ralentissement de l'écoulement des eaux. Ce qui a pour conséquence l'augmentation de la concentration des matières issues des rejets urbains et industriels dans les eaux. La faible teneur de la DCO enregistrée après la baie d'Anna (E13) serait liée au fait que cette zone est éloignée des rejets de matières d'origines urbaine et industrielle. Les valeurs du rapport $\mathrm{DCO} \mathrm{DBO}_{5}(1,25$ à 1,97) montrent que les eaux de la lagune contiennent essentiellement des matières biodégradables en saison sèche. Ces matières proviennent d'une part des eaux de drainage des terres agricoles et d'autre part des effluents domestiques et industrielles. Les industries situées au bord de la lagune Ebrié interviennent dans quatre (4) domaines d'activités (textile, agro-alimentaire, chimie et mines, pétrole et énergie). Les déchets de ces industries sont déversés directement dans la lagune sans aucun traitement préalable. Les plus importantes d'entre elles sont par ordre d'importance : l'industrie agro-alimentaire, de la chimie et l'industrie des mines, pétrole et énergie (Aka, 2017).

\section{$\checkmark \quad$ Etude des corrélations entre les paramètres physico-chimiques}

La bonne corrélation entre la $\mathrm{DCO}$ et de la $\mathrm{DBO}_{5}$, en saison sèche et en saison pluvieuse, traduit que ces deux paramètres évoluent dans le même sens. Concernant la bonne corrélation entre la salinité et le nitrite, elle pourrait indiquer l'influence des eaux océaniques sur ce nutriment (Inza et al, 2009). Cette observation est accord avec celle obtenue dans la lagune de GrandLahou. Dans cette lagune une corrélation très significative $(\mathrm{r}=0,86)$ a été également observée entre la salinité et le nitrite (Konan et al, 2013).La salinité et la température ont une bonne anti-corrélation en saison sèche et en saison pluvieuse. Cette relation témoigne d'une évolution contraire entre ces deux paramètres. Une baisse de la température entrainerait donc une augmentation de la salinité et vice-versa. La corrélation nulle entre la DCO et le pH en saison pluvieuse et en saison sèche indiquerait que l'évolution des teneurs de la DCO ne dépend pas de l'évolution des valeurs du $\mathrm{pH}$.

\section{Conclusion}

La variation des matières oxydables est fonction de l'alternance de l'abondance des eaux continentales et des eaux marines dans la lagune. Il existe deux groupes parmi les paramètres étudiés. Le groupe un (1) est le groupe des paramètres dont les valeurs sont fortes en période pluvieuse. Ce sont l'oxygène dissous, le nitrite, le nitrate, l'orthophosphate, la demande chimique en oxygène et la demande biochimique en oxygène. Le groupe deux (2) est celui des paramètres ayant une forte valeur en période sèche. Il s'agit du $\mathrm{pH}$, de la température, de la salinité et de l'ammonium. Les baies 
enregistrent les plus fortes teneurs des matières oxydables. La lagune Ebrié contient en période pluvieuse des substances biodégradables, des substances difficilement biodégradables et des substances non biodégradables. En période sèche, elle contient uniquement des substances biodégradables. Les eaux lagunaires reçoivent plus de matières oxydables dans les zones fortement urbanisées que dans les zones peu ou non urbanisées. Pour la préservation de cette lagune, les autorités compétentes doivent penser à la réhabilitation des stations d'épurations.

\section{Remerciements}

Nous exprimons nos sincères remerciements au Département des Géosciences Marines (DGM) de l'Université Félix Houphouët-Boigny de Cocody-Abidjan et au Centre de Recherche Océanologique (Côte d'Ivoire) pour leur contribution à la réalisation de ce travail.

\section{References:}

1. Adingra A.A. et Kouassi A.M., 2011. Pollution en lagune Ebrié et ses impacts sur l'environnement et les populations riveraines. F. Tech. et Doc. Vulg., pp48-53.

2. afnor (Association Française de Normalisation), 2001. Qualité de l'eau, éléments majeurs-autre éléments et composés minéraux. Aubenas Ardèche, $6^{\text {ème }}$ Edition, 635p.

3. Aka A.M., 2017. Qualité environnementale d'un estuaire à pression anthropique et à forçages naturels saisonniers en lagune Ebrié (Sud-Est de la Côte d'Ivoire). Thèse de Doctorat, Université Félix HouphouëtBoigny de Cocody, Abidjan, 243p.

4. Aka K. et Koné M., 1996. Les lagunes d'Abidjan asphyxiées par la pollution. Africa Geoscience Review, 3(3-4), pp 461-470.

5. Aka A.M., Wognin A.V., Amani E.M. et Irié B.T.J.G., Coulibaly A.S., Monde S., 2017a.

6. Caractérisation saisonnière de l'hydrologie d'un estuaire sous forte pression anthropique et naturelle : la lagune Ebrié (Sud-Est de la Côte d'Ivoire). Journal of Environmental Hydrology, 3(8), pp1-12.

7. Aka A.M., Wognin A.V., Amani E.M. et Irié B.T.J.G., Coulibaly A.S., Monde S., 2017b. Analyse des paramètres physico-chimiques et bactériologiques des eaux de l'estuaire de la lagune Ebrié (Sud-Est de la Côte d'Ivoire). European Journal of Scientific Research, 147(3), pp301-314.

8. Kouassi A.M., TidouA.S. et KamenanA., 2005. Caractéristiques hydrochimiques et microbiologiques des eaux de la lagune Ebrié (Côte d'Ivoire). Partie I : Variabilité saisonnière des paramètres hydrochimiques. Agronomie Africaine, 17 (2), pp117-136. 
9. Bamba S. B., Ouffoue S., Ble M. C., Metongo S. B. et Bakayoko S., 2008. Etatde l'environnement lagunaire de l'Île Vitré (Grand-Bassam, Côte d'Ivoire) : aspects physiques,chimiques et biologiques. Rev. Ivoir. Sci. Technol., 12, pp77- 92.

10. Binder E., 1968. Répartition des mollusques dans la lagune Ébrié (Côte d'Ivoire). Cahier de 1'O.R.S.T.O.M., Série Hydrobiologie, II (3-4), pp1-34.

11. Dufour P., 1982. Les frontiers naturelles et humaines du système lagunaire Ebrié. Incidence surl'hydroclimat. Hydrobiologia 94, pp105120.

12. Durand J.R. et Guiral D., 1994. Hydroclimat et hydrochimie. in Environnement et ressources aquatiques de Côte d'Ivoire : tome 2, les milieux lagunaires. Ed. ORSTOM, pp59-90.

13. Hammami J., Brahim M. et Gueddari M., 2005.Essai d'évaluation de la qualité des eaux de ruissellement du bassin versant de la lagune de Bizerte. Bull. Inst. Natn. Scien. Tech. Mer de Salammbô, 32, pp69-77.

14. Hernandez I., Hwang S.J. et Heath R.T., 1996. Measurement of phosphomonoesterase activity with a radiolabelled glucose-6phosphate. Role in the phosphorus requirement of phytoplankton and bacterioplankton in a temperate mesotrophic lake. Arch. Hydrobiol., 137, pp265-280.

15. Inza B., Metongo B.S., Assoi O.E, Trokourey A. et Yobou B., 2009. Caractérisation physico-chimique des eaux et des sédiments de surface de la baie des milliardaires, lagune Ebrié, Côte d'Ivoire. Rev. Ivoir. Sci. Technol., 131, pp35-154.

16. Irié B.T.J.-G., 2017. Caractérisation de l'interface eau-sédiment dans un environnement lagunaire à forçage : hydrologie et évaluation environnementale du chenal est de la lagune Ebrié. Thèse de Doctorat, Université Félix Houphouët-Boigny de Cocody, Abidjan, 201p.

17. Konan K.S., Kouassi K.L., KouaméK.I., KouassiA.M.et Gnakri D., 2013. Hydrologie et hydrochimie des eaux dans la zone de construction du chenal duport de pêche de Grand-Lahou, Côte d'Ivoire.Int. J. Biol. Chem. Sci. 7(2), pp819-831.

18. Lacroix S., 2006. Statistiques. Consulté le 21/09/2010. URL : www.cableamos.com/ sylvain.lacroix /maths/526-536/Statistique.

19. Makhoukh M., Sbaa M., Berrahou A. et Van C.M., 2011. Contribution à l'étude physico-chimique des eaux superficielles de l'Oued Moulouya (Maroc oriental). Larh. Journ., 09, pp149-169.

20. Pages J., Lemasson L. et Dufour P., 1979. Eléments nutritifs et production primaire dansles lagunes de Côte d'Ivoire. Cycle annuel. Arch. Sci. Centre Rech. Océanogr. Abidjan, 5, pp1-60. 
21. Rodier J., Legube B., Merlet N., Rebouillon P., Moulin L., Chomode P., Dujardin P., Gosselin S., Seux R. et Mardini A.F., 2009. L'analyse de l'eau, $9^{\text {ème }}$ édition, Dunod, $1511 \mathrm{p}$.

22. Shaher D., Malka K. et Yoram A., 1993. Nitrification pattern in a fluctuating anaerobic-aerobic pond environnement. Wat. Ress., 27 (9), Pergamson, pp1469-1475.

23. Tastet J.P., 1974. L'environnement physique du système lagunaire Ebrié. Sériedocumentation, 11, 28p.

24. Yapo B.O., Mambo V., Seka A., Yapi A.D. et Houenou P., 2009. Caractérisation par fractionnement gravimétrique de la matière organique contenue dans les eaux usées : application à l'étude de la biodégradabilité. Journal de la Société Ouest-Africaine de Chimie, 27, pp21-37. 